\title{
Emerging biomarkers in the diagnosis of prostate cancer
}

\section{Xavier Filella \\ Esther Fernández-Galan \\ Rosa Fernández Bonifacio \\ Laura Foj}

Department of Biochemistry and Molecular Genetics (CDB), Hospital Clínic, IDIBAPS, Barcelona, Catalonia, Spain

Correspondence: Xavier Filella Department of Biochemistry and Molecular Genetics (CDB), Hospital Clínic, C/ Villarroel, I70, Barcelona,

Catalonia 08036, Spain

Tel +34932279375

Fax +34932279376

Email xfilella@clinic.cat
This article was published in the following Dove Press journal: Pharmacogenomics and Personalized Medicine

\begin{abstract}
Prostate cancer (PCa) is the second most common cancer in men worldwide. A large proportion of $\mathrm{PCa}$ are latent, never destined to progress or affect the patients' life. It is of utmost importance to identify which PCa are destined to progress and which would benefit from an early radical treatment. Prostate-specific antigen (PSA) remains the most used test to detect PCa. Its limited specificity and an elevated rate of overdiagnosis are the main problems associated with PSA testing. New PCa biomarkers have been proposed to improve the accuracy of PSA in the management of early PCa. Commercially available biomarkers such as PCA3 score, Prostate Health Index (PHI), and the four-kallikrein panel are used with the purpose of reducing the number of unnecessary biopsies and providing information related to the aggressiveness of the tumor. The relationship with PCa aggressiveness seems to be confirmed by PHI and the fourkallikrein panel, but not by the PCA3 score. In this review, we also summarize new promising biomarkers, such as PSA glycoforms, TMPRSS2:ERG fusion gene, microRNAs, circulating tumor cells, androgen receptor variants, and PTEN gene. All these emerging biomarkers could change the management of early $\mathrm{PCa}$, offering more accurate results than PSA. Nonetheless, large prospective studies comparing these new biomarkers among them are required to know their real value in $\mathrm{PCa}$ detection and prognosis.
\end{abstract}

Keywords: prostate cancer, PSA, PHI, four-kallikrein panel, PCA3, miRNAs

\section{Introduction}

Prostate cancer ( $\mathrm{PCa}$ ) is the second most common cancer in men worldwide, with an estimated incidence of 1.1 million new cases in $2012 .{ }^{1}$ The highest rate of incidence has been observed in western countries, at least partially due to the widespread use of prostate-specific antigen (PSA) testing. Thus, in the USA, PCa is the most frequent tumor, with 1,64,690 new estimated cases for $2018 .^{2}$ Actually, a large proportion of $\mathrm{PCa}$ are latent, never destined to progress or affect the patients' life. Klotz ${ }^{3}$ has estimated that the percentage of patients with a low risk of progression is between $50 \%$ and $60 \%$ of newly diagnosed cases. Thus, the overdiagnosis of indolent tumors is a major problem associated with $\mathrm{PCa}$ early diagnosis.

In fact, $\mathrm{PCa}$ is a very heterogeneous disease, with abysmal differences in clinical evolution, ranging from clinically insignificant tumors to lethal castration-resistant $\mathrm{PCa}$ (CRPC). Because of the side effects of treatments used in $\mathrm{PCa}$, it is critical to select the patients for whom active treatment is required. Active surveillance has been proposed to reduce the negative effects of overdiagnosis and overtreatment, delaying any kind of definitive treatment and applying it only if there is evidence of progression. 
Patients with biopsy Gleason score 6, PSA $<10 \mu \mathrm{g} / \mathrm{L}$, and a low percentage of biopsy material involved with cancer are candidates for active surveillance. ${ }^{4}$

Different risk classification tools have been developed to distinguish patients with early PCa according to the prognosis, including the D'Amico classification system, ${ }^{5}$ the Cancer of the Prostate Risk Assessment score, ${ }^{6}$ and the National Comprehensive Cancer Network (NCCN) risk groups classification. ${ }^{7}$ Table 1 describes the different risk classification systems. All these systems recognize a low risk of progression for patients with a biopsy Gleason score $\leq 6$. Actually, several authors suggest that Gleason score 6 disease should not be labeled as cancer because these tumors have an extremely low potential of metastasis, although they have the hallmarks of cancer from a pathological perspective. ${ }^{8}$

Table I Risk classification systems of PCa

\begin{tabular}{|c|c|c|}
\hline \multicolumn{3}{|l|}{ D'Amico classification $^{5}$} \\
\hline Low risk & \multicolumn{2}{|c|}{$\begin{array}{l}\text { Gleason score } \leq 6, \text { PSA }<10 \mu g / L \text {, clinical } \\
\text { stage } \leq T 2 a\end{array}$} \\
\hline Intermediate risk & \multicolumn{2}{|c|}{$\begin{array}{l}\text { Gleason score } \leq 7 \text {, PSA } 10-20 \mu g / L \text {, clinical } \\
\text { stage } \leq T 2 b\end{array}$} \\
\hline High risk & \multicolumn{2}{|c|}{$\begin{array}{l}\text { Gleason score }>7, \text { PSA }>20 \mu g / L \text {, clinical } \\
\text { stage } T 2 c\end{array}$} \\
\hline \multicolumn{3}{|l|}{$\overline{\text { CAPRA score }}{ }^{*, 6}$} \\
\hline & Level & Points \\
\hline \multirow[t]{5}{*}{ PSA at diagnosis } & $2-6.0 \mu g / L$ & 0 \\
\hline & $6.1-10 \mu g / L$ & I \\
\hline & $10.1-20 \mu g / L$ & 2 \\
\hline & $20.1-30 \mu g / L$ & 3 \\
\hline & $>30 \mu g / L$ & 4 \\
\hline \multirow[t]{2}{*}{ Age at diagnosis } & $<50$ years old & 0 \\
\hline & $\geq 50$ years old & I \\
\hline \multirow[t]{2}{*}{ T stage } & $\mathrm{TI} / \mathrm{T} 2$ & 0 \\
\hline & T3 & I \\
\hline \multirow{2}{*}{$\begin{array}{l}\text { Percent of biopsy cores } \\
\text { involved with cancer }\end{array}$} & $<34 \%$ & 0 \\
\hline & $\geq 34 \%$ & I \\
\hline \multicolumn{3}{|l|}{$\overline{\text { NCCN classification }}{ }^{7}$} \\
\hline Very low risk & \multicolumn{2}{|c|}{$\begin{array}{l}\text { Gleason score } \leq 6, \mathrm{PSA}<10 \mu \mathrm{g} / \mathrm{L} \text {, Stage } \mathrm{TI} \mathrm{c} \text {, } \\
\text { not more than two cores with cancer, } \\
<50 \% \text { of core involved with cancer, and PSA } \\
\text { density }<0.15\end{array}$} \\
\hline Low risk & \multicolumn{2}{|l|}{$\begin{array}{l}\text { Gleason score } \leq \\
\text { or } \mathrm{T} 2 \mathrm{a}\end{array}$} \\
\hline Intermediate risk & \multicolumn{2}{|c|}{ Gleason score 7, PSA I0-20 $\mu \mathrm{g} / \mathrm{L}$, Stage } \\
\hline High risk & \multicolumn{2}{|c|}{ Gleason score $\geq 8, \mathrm{PSA} \geq 20 \mu \mathrm{g} / \mathrm{L}$, Stage T3a } \\
\hline
\end{tabular}

Note: *The CAPRA score is the sum of the points assigned for each variable and classifies patients into low, intermediate, and high risk. A CAPRA score of 0 to 2 indicates low-risk; a CAPRA score of 3 to 5 indicates intermediate-risk; and a CAPRA score from 6 to 10 indicates high-risk.

Abbreviations: CAPRA, Cancer of the Prostate Risk Assessment; NCCN, National Comprehensive Cancer Network; PCa, prostate cancer; PSA, prostatespecific antigen.
Understanding the biological bases of the clinical heterogeneity of $\mathrm{PCa}$ is one of the greatest challenges for the best management of PCa patients. Several recent studies have investigated the molecular bases of $\mathrm{PCa}$ and have drawn attention to the remarkable heterogeneity of this tumor. Rubin et $\mathrm{al}^{9}$ showed a significant frequency increase in genomic amplifications and deletions ( $p: 0.013)$ and in nonsynonymous point mutations ( $p: 0.008)$ with increasing Gleason score in a group of 426 clinically localized PCa patients treated with radical prostatectomy. On the other hand, Lapointe et $\mathrm{al}^{10}$ using cDNA microarrays containing 26,000 genes distinguished three different molecular subtypes of $\mathrm{PCa}$, suggesting a basis for improved prognostication and treatment stratification. More recently, the researchers of the Cancer Genome Atlas Research Network ${ }^{11}$ identified seven different molecular subtypes, remarking PCa heterogeneity. This study described a molecular taxonomy in which $74 \%$ of $\mathrm{PCa}$ were classified in one of seven subtypes defined by ERG fusions (46\%), ETV1/ETV4/FLI1 fusions or overexpression $(8 \%, 4 \%, 1 \%$, respectively), or by SPOP $(11 \%)$, FOXA1 (3\%), and IDH1 (1\%) mutations.

In this review, we summarize the recent progress regarding the new emerging biomarkers related to $\mathrm{PCa}$ in the era of personalized medicine. Table 2 shows a summary of the commercially available blood and urine biomarkers used in clinical practice.

Many of these biomarkers also provide valuable prognostic information that could have important implications for the adequate selection of patients for active surveillance or for radical treatment. Furthermore, advances in genomic techniques have allowed the description of new promising PCa biomarkers, such as TMPRSS2:ERG fusion gene, micro RNAs (miRNAs), and circulating tumor cells (CTCs), PTEN. On the other hand, several tissue biomarkers, among them the commercially available Prolaris ${ }^{\circledR}$ and Oncotype $\mathrm{DX}^{\circledR}$ tests, have been proposed to provide an accurate assessment of cancer aggressiveness. Finally, androgen receptor splice variant-7 (AR-V7) is useful to select the treatment in patients with advanced $\mathrm{PCa}$. Figure 1 shows an overview of $\mathrm{PCa}$ biomarkers according to the test's clinical utility.

\section{PSA-derived PCa biomarkers Prostate Health Index (PHI)}

PSA, also known as human kallikrein 3, is a glycoprotein that circulates into the blood bound to protease inhibitors, among them $\alpha 1$-antichymotrypsin and $\alpha 2$-macroglobulin. Only a small amount is not protein bound and is called free PSA (fPSA). The percentage of fPSA to total PSA (\% $\%$ PSA) 
Table 2 Emerging PCa biomarkers commercially available

\begin{tabular}{|c|c|c|c|c|c|}
\hline Panel & Manufacturer & Specimen & Description & Clinical usefulness & $\begin{array}{l}\text { Approved } \\
\text { by FDA }\end{array}$ \\
\hline $\mathrm{PHI}^{13,14}$ & $\begin{array}{l}\text { Beckman } \\
\text { Coulter }\end{array}$ & Serum & $\begin{array}{l}\text { Measures total PSA, fPSA, } \\
\text { and p2PSA }\end{array}$ & PCa detection and prognosis & Yes \\
\hline $\begin{array}{l}\text { Four-kallikrein panel } \\
(4 \mathrm{Kscore})^{19}\end{array}$ & Opko & Serum or plasma & $\begin{array}{l}\text { Measures total PSA, fPSA, } \\
\text { iPSA, and hK2 }\end{array}$ & PCa detection and prognosis & No* \\
\hline $\mathrm{PCA}^{32}$ & Hologic & $\begin{array}{l}\text { Urine collected after } \\
\text { prostate massage }\end{array}$ & $\begin{array}{l}\text { Measures mRNA PCA3 in } \\
\text { relation to mRNA PSA }\end{array}$ & $\begin{array}{l}\text { PCa detection. Contradictory } \\
\text { results concerning prognosis }\end{array}$ & Yes \\
\hline $\begin{array}{l}\text { Circulating tumor cells } \\
\left.\text { (CellSearch }^{\circledR} \text { platform }\right)^{66}\end{array}$ & $\begin{array}{l}\text { Janssen } \\
\text { Diagnostics }\end{array}$ & Whole blood & $\begin{array}{l}\text { Measures circulating tumor } \\
\text { cells on CellSearch platform }\end{array}$ & $\begin{array}{l}\text { Associated with decreased } \\
\text { overall survival in metastatic } \mathrm{PCa}\end{array}$ & Yes \\
\hline $\begin{array}{l}\text { OncotypeDX }{ }^{\circledR} \text { AR-V7 } \\
\text { Nucleus Detect }{ }^{75,77}\end{array}$ & Epic Sciences & Whole blood & $\begin{array}{l}\text { Measures expression of } \\
\text { AR-V7 in the nucleus of } \\
\text { circulating tumor cells }\end{array}$ & $\begin{array}{l}\text { To select treatment for CRPC } \\
\text { patients }\end{array}$ & No* \\
\hline
\end{tabular}

Note: *These biomarkers are available from CLIA-certified clinical laboratories.

Abbreviations: AR-V7, androgen receptor splice variant-7; CLIA, Clinical Laboratory Improvement Amendment; CRPC, castration-resistant PCa; FDA, US Food and Drug Administration; fPSA, free PSA; hK2, human kallikrein 2; iPSA, intact PSA; PCa, prostate cancer; PCA3, prostate cancer gene 3; PHI, Prostate Health Index; PSA, prostatespecific antigen.

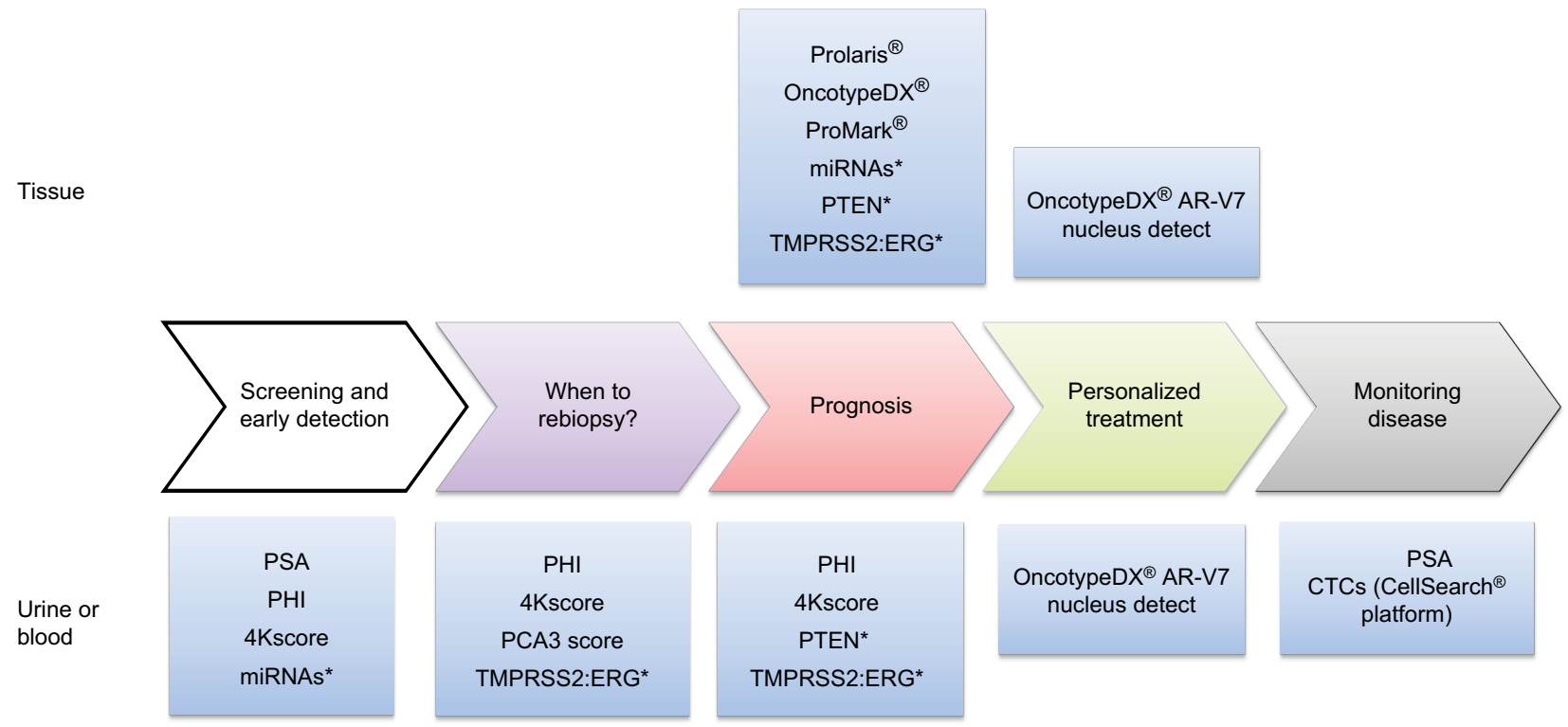

Figure I Overview of PCa biomarkers according to the test's clinical utility.

Note: *Biomarker in evaluation.

Abbreviations: AR-V7, androgen receptor splice variant-7; CTCs, circulating tumor cells; PCa, prostate cancer; PCA3, prostate cancer gene 3; PHI, Prostate Health Index; PSA, prostate-specific antigen.

is significantly decreased in patients with $\mathrm{PCa}$, although an overlap of results is observed comparing patients with and without PCa. Thus, the clinical interpretation of \% fPSA results is complicated, and probably this test only provides additional information in the decision to perform prostate biopsies when levels reach extreme values. ${ }^{12}$ Besides \%fPSA, other PSA derivatives have been proposed as PCa biomarkers, including PSA-based nomograms, with the aim to reduce the number of unnecessary prostate biopsies (Table 3 ).

More recently, fPSA have been found to include the isoforms benign PSA, intact PSA (iPSA) and proPSA, a precursor form of PSA (Figure 2). Initial results obtained about proPSA suggested its value in the detection of PCa. Currently, the [-2]proPSA, the most stable form of proPSA, could be measured using an automated immunoassay developed by Beckman Coulter, called p2PSA.

The PHI combines total PSA, PPSA, and p2PSA, according to the formula (p2PSA/fPSA)* $\sqrt{ }$ total PSA. The test was approved by the US Food and Drug Administration (FDA) in June 2012 for the detection of PCa in men $>50$ years, PSA between 4 and $10 \mu \mathrm{g} / \mathrm{L}$, and a nonsuspicious digital rectal examination (DRE). 
Table 3 Description of PSA derivatives proposed for PCa detection

\begin{tabular}{|c|c|}
\hline Test biomarker & Description \\
\hline \%fPSA & Percentage of free PSA to total PSA \\
\hline $\begin{array}{l}\text { PSA-specific } \\
\text { reference ranges }\end{array}$ & fic reference limits for serum PSA \\
\hline PSA velocity & $\begin{array}{l}\text { The rate of rise of the PSA over time, given in } \\
\mu \mathrm{g} / \mathrm{L} / \mathrm{year}\end{array}$ \\
\hline PSA doubling time & Number of months it takes for the PSA to double \\
\hline PSA density & $\begin{array}{l}\text { Quotient between PSA serum levels and the } \\
\text { volume of the prostate }\end{array}$ \\
\hline Complexed PSA & $\begin{array}{l}\text { PSA bound to } \alpha \mathrm{I} \text {-antichymotrypsin, measured with } \\
\text { the assay Advia Centaur complexed PSA }\end{array}$ \\
\hline $\begin{array}{l}\text { PSA-based } \\
\text { nomograms }\end{array}$ & $\begin{array}{l}\text { Graphical representations of a multivariate logistic } \\
\text { regression analysis based on demographic, clinical, } \\
\text { and biochemical variables, including age of the } \\
\text { patient, family history of PCa, DRE, prostate } \\
\text { volume, and PSA serum levels }\end{array}$ \\
\hline PSA glycoforms & $\begin{array}{l}\text { Changes in PSA glycosylation patterns in serum } \\
\text { related to PCa }\end{array}$ \\
\hline
\end{tabular}

Abbreviations: DRE, digital rectal examination; fPSA, free PSA; PCa, prostate cancer; PSA, prostate-specific antigen.
PHI demonstrated a higher accuracy than total PSA and \% fPSA to predict the presence of PCa at biopsy, showing area under curves (AUCs) from 0.703 to 0.77 according to a meta-analysis published in $2013 .{ }^{13}$ Similar results have been shown by a recent meta-analysis evaluating PHI in men with PSA from 2 to $10 \mu \mathrm{g} / \mathrm{L}$ at first biopsy, reporting AUCs of 0.74 and 0.63 for PHI and \%fPSA, respectively. ${ }^{14}$ In our experience, PHI outperforms \%fPSA, obtaining AUCs of 0.748 and 0.70 , respectively. On the other hand, we found a relationship between PHI and prostate volume, obtaining AUCs of $0.818,0.716$, and 0.654 for patients with a prostate volume of $\leq 35,36-50$, and $>50$, respectively. ${ }^{15}$ In this regard, Tosoian et $\mathrm{al}^{16}$ recently underlined the value of prostate volume in the interpretation of PHI values. According to this group, PHI density has a higher discriminative ability to detect clinically significant $\mathrm{PCa}$ (AUC 0.84) than PHI (AUC 0.76 ) or \%fPSA (0.75).

Furthermore, PHI showed an association with the aggressiveness of the tumor, with higher levels in patients

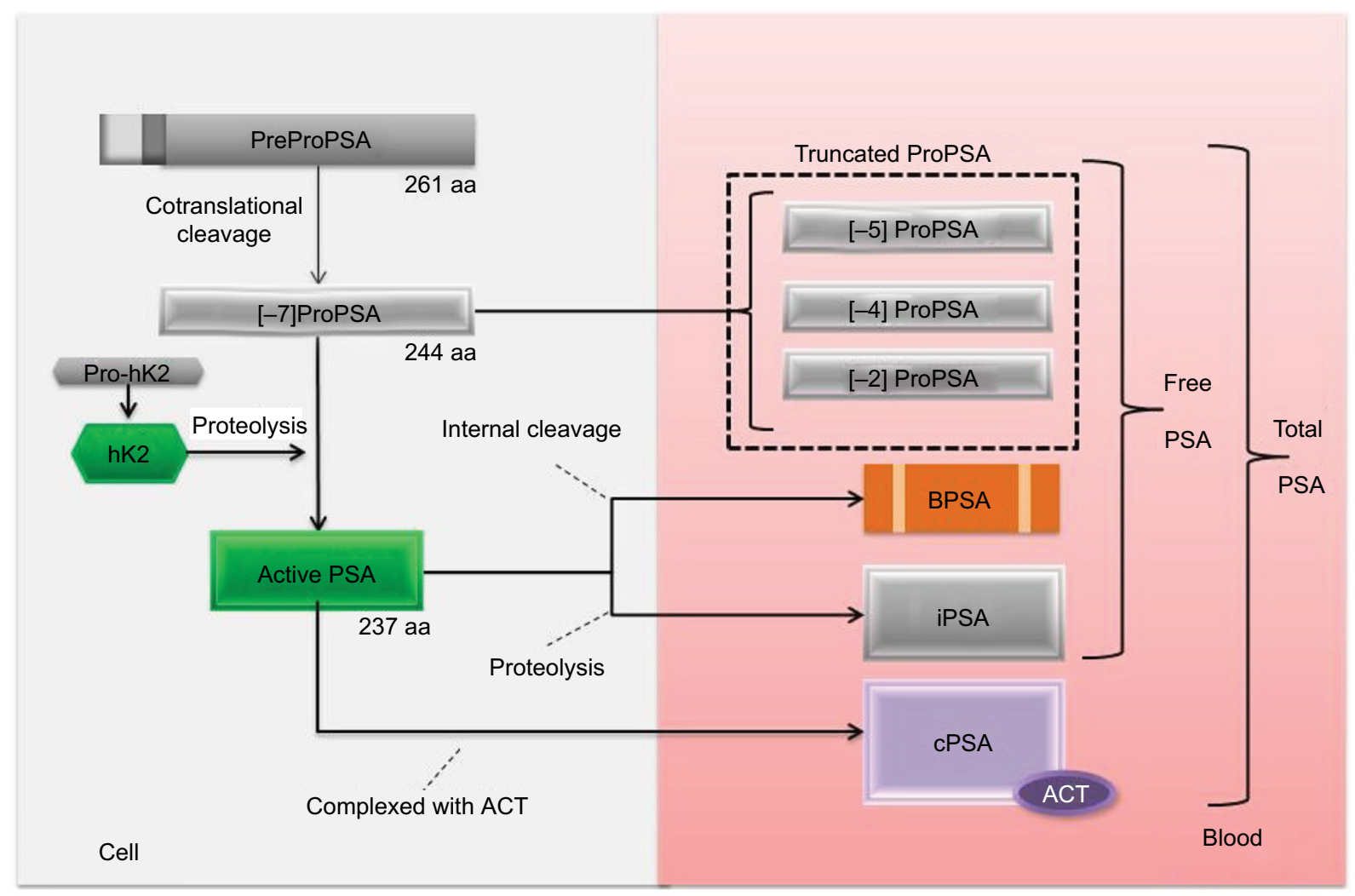

Figure 2 Molecular forms of PSA.

Notes: In serum, PSA (80\%-95\%) circulates mainly as a complexed form (cPSA) bound to several protease inhibitors (e.g., $\alpha$ I anti-chymotrypsin and $\alpha 2$ macroglobulin). Free PSA is a small fraction of total PSA, which is also composed by three different subfractions: BPSA, iPSA, and proPSA. The native form of proPSA is [-7] proPSA, which contains a 7-amino acid $\mathrm{N}$-terminal pro-leader peptide. Through the proteolytic cleavage of this peptide, promoted by the kallikrein hK2, the other truncated forms of proPSA, known as [-2], [-4], and [-5] proPSA, are produced.

Abbreviations: ACT, alpha I-antichymotrypsin; BPSA, benign PSA; cPSA, complexed PSA; hK2, human kallikrein 2; iPSA, intact PSA; PSA, prostate-specific antigen. 
with aggressive PCa. According to our results, PHI was significantly higher in patients with a Gleason score $\geq 7$ than in patients with Gleason score 6 . Besides, we found significant values for $\mathrm{PHI}$ in patients with clinical stage T2-T3 than in patients with stage T1c. ${ }^{17}$ On this matter, De la Calle et al ${ }^{18}$ recently underlined that higher $\mathrm{PHI}$ values were significantly associated with a Gleason score $\geq 7$, reporting an AUC of 0.815 to detect aggressive PCa. Furthermore, the authors reported that $\mathrm{PHI}$ has a higher specificity $(36 \%)$ to detect aggressive PCa than total PSA (17\%) and \%fPSA (19\%) at $95 \%$ sensitivity.

\section{Four-kallikrein panel}

Several studies remark the usefulness of the four-kallikrein panel in the detection and prognosis of PCa. The panel comprises total PSA, fPSA, iPSA, and human kallikrein 2, a glycoprotein with high homology to PSA. The four-kallikrein panel outperforms total PSA in predicting the outcome of a prostate biopsy in several cohorts of men enrolled in screening studies, showing AUCs from 0.674 to 0.832 . Furthermore, adding DRE, the panel demonstrated an excellent diagnostic performance for high-grade $\mathrm{PCa}$ (Gleason score $\geq 7$ ), showing AUCs from 0.798 to 0.873 in previously screened men and from 0.837 to 0.903 in unscreened men. ${ }^{19}$

This panel has been commercialized by Opko Diagnostics with the name of $4 \mathrm{Kscore}$. The test combines the four-kallikrein panel together with patient age, DRE, and the history of prior biopsy in an algorithm that calculates the individual patient's risk of high-grade PCa. Thus, the aim of the test is to reduce the overdetection of $\mathrm{PCa}$, offering biopsy only to those patients with an elevated risk of high-grade $\mathrm{PCa}$. A multi-institutional prospective trial developed in the USA, evaluating 1012 men undergoing a prostate biopsy, confirmed the value of 4 Kscore. The authors documented that the test could discriminate between patients with and without Gleason score $\geq 7$ with an AUC of 0.821 . The authors indicated that $30 \%$ of biopsies could be avoided using a cutoff value of $6 \%$, delaying diagnosis for $1.3 \%$ of high-grade PCa patients. ${ }^{20}$ Similar results have been found by Braun et $\mathrm{al}^{21}$ in a cohort of 749 men submitted to biopsy based on total PSA $\geq 3.0 \mu \mathrm{g} / \mathrm{L}, \%$ fPSA $\leq 20 \%$, or a suspicious DRE. According to this study, the four-kallikrein panel can reduce the number of men requiring biopsy. Therefore, $17 \%$ of biopsies could be avoided using a threshold of $6 \%$ risk of high-grade $\mathrm{PCa}$, whereas diagnosis would be delayed for 8 of 208 (3.8\%) patients with high-grade PCa. Furthermore, the authors showed an AUC of 0.784 for a model including age, the four-kallikrein panel, and DRE.
Additionally, the test has been able to predict the longterm development of distant metastasis. Results published by Stattin et $\mathrm{al}^{22}$ showed that $4 \mathrm{Kscore}$ measured at 50 and 60 years old provides risk stratification of patients, classifying patients into two groups according to the probability of developing distant metastasis 20 years later. The authors concluded that the four-kallikrein panel could aid biopsy decision making in patients with a modest PSA elevation in midlife.

A recent study published by Lin et $\mathrm{al}^{23}$ evaluated the ability of $4 \mathrm{Kscore}$ to predict the presence of high-grade $\mathrm{PCa}$ in 718 men enrolled on active surveillance in nine centers. They showed that a clinical model including $4 \mathrm{Kscore}$ significantly ( $p$ : 0.043 ) improved the accuracy to predict reclassification compared with a clinical model including PSA at the first surveillance biopsy, showing AUCs of 0.783 and 0.740 , respectively. However, no differences between both models were obtained when the prediction of reclassification at subsequent biopsies was studied (AUC 0.754 vs. 0.755).

\section{PSA glycoforms}

PSA, like most serum tumor markers, is a glycoprotein. Specific changes in PSA glycosylation patterns have been described in LNCaP tumor cells. ${ }^{24}$ Further studies demonstrated that these differences are also measurable in serum, showing different glycosylation patterns between $\mathrm{PCa}$ patients and healthy subjects. ${ }^{25}$ Based on existing literature, the most significant alterations found in PSA molecule were in $\alpha 2-3$-linked sialic acid and core fucosylation.

Significant increase of $\alpha 2,3$-sialic acid in PCa patients compared with benign prostatic hyperplasia $(\mathrm{BPH})$ patients have been reported by Ohyama et $\mathrm{al}^{26}$ More recently, Llop et $\mathrm{al}^{27}$ underlined that the serum $\alpha 2,3$-sialic acid percentage of PSA also identifies aggressive $\mathrm{PCa}$ and correlates with Gleason score. The cufoff value of $30 \%$ differentiates between high-risk $\mathrm{PCa}$ and the groups of $\mathrm{BPH}$, low- and intermediate-risk $\mathrm{PCa}$ with a sensitivity and specificity of $85.7 \%$ and $95.5 \%$, respectively. The AUC obtained for this test was higher (0.966) than the AUC obtained for total PSA (0.865) and \%fPSA (0.562).

Several authors reported a significant increase of PSA fucosylation in PCa patients in comparison to healthy controls $^{28}$ together with a significant correlation with Gleason score. ${ }^{29}$ However, a decrease in core fucosylation levels in PSA from high-risk PCa compared with BPH patients was detected in other study. ${ }^{27}$

Nowadays, glycoproteomic analysis still remains a challenge. Comparison between independent studies is 
very difficult because of the large number of techniques used and the lack of a reference method. Most authors use lectin-based methodologies, but other analytical techniques such as high-performance liquid chromatography, capillary electrophoresis, and mass spectrometry have been used alone or in combination. The future application of glycosylation PSA analysis to clinical practice could offer additional tools to improve accuracy of PCa diagnosis. More studies are needed for validating these promising results before clinical implementation.

\section{Molecular biomarkers PCA3}

Several studies have suggested that a large number of long noncoding RNAs are functional and, through regulatory mechanisms, are involved in carcinogenesis processes. Recently, several long noncoding RNAs have been reported to be deregulated in $\mathrm{PCa}$, including PCGEM, PCAT-1, and the prostate cancer gene 3 (PCA3).

The $P C A 3$ gene, initially called Differential Display Code 3, was identified in 1999 by Bussemakers et $\mathrm{al}^{30}$ who described a strong overexpression of $P C A 3$ gene in prostatic tumors compared with normal prostate tissue. The gene encoding PCA3 is located on chromosome 9q21-22 in antisense orientation and consists of four exons and three introns. There is an alternative polyadenylation at three different sites of exon 4 ( $4 \mathrm{a}, 4 \mathrm{~b}$, and $4 \mathrm{c}$ ) yielding three transcripts of different sizes. Additional studies described four extra transcription start sites and two new differentially spliced exons (2a and 2b). PCA3 gene is inserted in the intron of a second gene, PRUNE2 or BMCC1, which is implicated in the control of oncogenic transformation, and it has been proposed that $P C A 3$ regulates $P R U N E 2$ levels through the formation of a double-stranded RNA. ${ }^{31}$

The mRNA PCA3 is measured using quantitative realtime polymerase chain reaction (qRT-PCR) in a urine sample obtained after a prostate massage in order to obtain the maximum amount of prostatic cells. This measurement must be performed simultaneously with the mRNA of PSA gene, which has a similar expression in cancerous and benign cells. Thus, a PCA3 score based on the ratio of PCA3 mRNA to PSA mRNA can be determined. The Progensa PCA3 test, currently commercialized by Hologic, is a semiautomated assay that includes isolation, amplification, hybridization, and quantification of PCA3 and PSA mRNAs using the DTS systems.

The PCA3 test obtained the Conformité européenne in 2006 and was approved by the FDA in 2012 to decide the repetition of the prostate biopsy in men $\geq 50$ years of age who have had one or more previous negative prostate biopsies. Several studies have evaluated the PCA3 discrimination ability describing their sensitivity and specificity, using ROC curve analysis and multivariate logistic regression models. These studies showed that PCA3 score outperforms PSA and \%fPSA. ${ }^{32}$ According to a meta-analysis recently published, the overall sensitivity, specificity, and AUC values were 0.63 , 0.88 , and 0.82 , respectively, for case-control studies, and $0.65,0.73$, and 0.75 , respectively, for prospective studies. ${ }^{33}$ This meta-analysis included 46 different studies, underlying that the cutoff of 35 was used in 26 institutions. However, the choice of the most appropriate cutoff for PCA3 score remains controversial, and the FDA suggests that a PCA3 score $<25$ is associated with a decreased probability of positive biopsy. In this regard, Roobol et $\mathrm{al}^{34}$ showed that the PCA3 sensitivity was $68 \%, 84 \%$, and $97 \%$, respectively, for the cutoffs of 35,20 , and 10 . Also, according to these authors, $26 \%$ of aggressive PCa were missed using the cutoff of 35 . On the other hand, the authors of this study remarked that false-positive results are an additional problem using PCA3 score, finding that $69 \%$ of patients with PCA3 score $\geq 100$ did not have a positive biopsy. A similar conclusion was reported by Schröder et al, ${ }^{35}$ although in this study significant efforts to detect a $\mathrm{PCa}$ were performed in patients with PCA3 score $\geq 100$.

The relationship of PCA3 score with $\mathrm{PCa}$ aggressiveness is controversial. Merola et $\mathrm{al}^{36}$ found a correlation between PCA3 and tumor aggressiveness, in terms of Gleason score, using a threshold of 51. These results are consistent with those reported by additional studies ${ }^{37,38}$; however, other authors disagree, showing that PCA3 is not correlated with Gleason score. ${ }^{39,40}$

\section{TMPRSS2:ERG fusion gene}

Genomic rearrangements are often observed in several hematologic malignancies and also in solid tumors, including PCa. ${ }^{41}$ The recent publication of the Cancer Genome Atlas molecular taxonomy of PCa identifies E26 transformationspecific (ETS) rearrangements as the most common subtype, involving $58 \%$ of tumors. ${ }^{11}$

The TMPRSS2:ERG gene rearrangements could be detected in urine samples obtained after a prostate massage using qRT-PCR. PSA mRNA is used for normalization and the ratio TMPRSS2:ERG mRNA/PSA mRNA is known as TMPRSS2:ERG score. The combination of the TMPRSS2:ERG and PCA3 scores has been proposed as a way to improve the prediction of the presence of $\mathrm{PCa}$ in the biopsy. A prospective multicenter study published by Leyten 
et $\mathrm{al}^{42}$ assessed the independent additional predictive value of TMPRSS2:ERG score and PCA3 score in relation to the European Randomized Study of Screening for Prostate Cancer (ERSPC) risk calculator in a cohort of 443 men. The authors reported that both scores significantly increased the predictive value obtained with the ERSPC risk calculator. The AUC of the ERSPC risk calculator increased from 0.799 to 0.842 when PCA3 and TMPRSS2:ERG scores were added. In addition, TMPRSS2:ERG score - but not PCA3 score - was associated with the biopsy Gleason score and the tumor clinical stage. The prospective study by Tomlins et $\mathrm{al}^{43}$ showed the value of the PCA3 and TMPRSS2:ERG scores when they were added to the Prostate Cancer Prevention Trial (PCPT) risk calculator based on PSA and several clinical variables. The AUCs increased from 0.639 for the PCPT risk calculator to 0.762 by adding both TMPRSS2:ERG and PCA3 scores. PCA3 and TMPRSS2:ERG scores were also evaluated to predict the high-risk PCa, with an AUC of 0.779 when both biomarkers were added.

Otherwise, McKiernan et $\mathrm{al}^{44}$ have developed the ExoDx Prostate IntelliScore urine exosome assay that measures PCA3, SPDEF, and ERG, obtaining a gene score. The test can differentiate (AUC 0.74) the patients with high-risk $\mathrm{PCa}$ from those with low-risk PCa and benign disease. The AUC increased to 0.77 when this score was added to other variables, such as PSA level, age, race, and family history. The authors concluded that this test improves the accuracy to detect high-risk PCa among men with elevated PSA levels, saving unnecessary biopsies.

\section{MicroRNAs}

While $90 \%$ of the genome is transcribed to mRNA, only $2 \%$ of this is finally translated into protein. The remaining $98 \%$ are noncoding RNA, including small nucleolar RNA, small interfering RNA, piwi-interacting RNA, long noncoding RNA, and miRNA. ${ }^{45}$ miRNAs are small ( $\approx 20$ nucleotides) and single-stranded RNAs. They act as posttranscriptional gene regulators by partial complementary base pairing to specific mRNAs. Our knowledge about these molecules has exponentially grown since the identification of the first miRNA, lin-4, in 1993 by Lee et al. ${ }^{46}$ Their involvement has been described in the control of very important cellular processes such as cell differentiation, proliferation, apoptosis, and also in carcinogenesis. Accordingly, in recent years, miRNAs have been extensively studied and proposed as potential biomarkers for many diseases, including cancer.

Different studies have shown an aberrant expression profile of miRNAs in several types of cancer including
PCa. Cumulative results show that miRNAs are involved in all steps of PCa development, such as cell proliferation, differentiation, and progression. For instance, the members of miR-34 family and let-7 family have been found involved in the regulation of cancer stem cells in $\mathrm{PCa} .{ }^{47,48}$ Other miRNAs regulate the apoptosis process. Thus, miR-21 is usually upregulated in $\mathrm{PCa}$, which results in a significantly reduced cell apoptosis mediated by its action on different targets like PTEN. ${ }^{49}$ Cellular apoptosis can also be blocked through the regulation of the antiapoptotic protein BCL2. BCL2 gene is a known target of several miRNAs, such as miR-15, miR-16, miR-205, and the miR-34 family. ${ }^{50-52}$

On the other hand, miRNAs are regulators of epithelialmesenchymal transition (EMT) and mesenchymal-epithelial transition (MET) ${ }^{53}$ events through the regulation of key proteins involved in these processes. ${ }^{54-56}$ Through the EMT process, an epithelial cell assumes a mesenchymal cell phenotype and exhibit proprieties like enhanced migratory capacity, invasiveness, and elevated resistance to apoptosis. This mechanism could be adopted by PCa tumor cells, thus, acquiring the ability to penetrate the walls of lymphatic or blood vessels to invade other tissues in the body. Subsequently, metastatic cells can reacquire epithelial characteristics, similar to cells in their corresponding primary tumor, through the MET.

Although accumulated evidences suggest that miRNAs are a powerful tool that could improve the diagnosis and management of $\mathrm{PCa}$, their clinical implementation depends on many factors. The usefulness of miRNAs depends directly on the ability to obtain quality-assured results and on the standardization of the measurement. This is why the accurate quantification of the miRNA has been one of the investigators' challenges during the last years. miRNAs can be hybridized to microarrays to identify specific profiles of different cancers. One of the most used techniques for validating miRNAs is qRT-PCR. Besides, digital PCR is a direct method for quantifying nucleic acids, particularly useful for samples with low amount of miRNAs, showing a high degree of sensitivity and precision compared with qRT-PCR.

In a previous review, ${ }^{57}$ we detected substantial differences among 30 studies evaluating the usefulness of circulating miRNAs as PCa biomarkers. We found that 74 miRNAs had been suggested as biomarker candidates, but only 25 of them were congruently associated with $\mathrm{PCa}$ in more than one study. In fact, only miR-141, miR-375, and miR-21 appeared repeatedly in more than 10 studies. Discordant results among studies could be related to methodological variations or differences in the cohorts. Several signatures 
have been proposed to increase accuracy obtained with PSA in PCa detection and prognosis. In this regard, Mihelich et $\mathrm{al}^{58}$ suggested a panel of 14 miRNAs to discriminate Gleason grade and to predict biochemical recurrence after radical prostatectomy. The authors developed an miR risk score that accurately classified a subset of patients with low risk of biochemical recurrence, which had a negative predictive value of 0.941. Similarly, Al-Qatati et $\mathrm{al}^{59}$ showed that miR-16, miR-148a, and miR-195 in plasma are significantly related to a Gleason score $\geq 8$. The authors concluded that these miRNAs may discriminate between intermediate- and high-risk Gleason scores, suggesting that their deregulation leads to PCa progression, resistance, and metastasis.

Other authors have suggested the analysis of miRNAs in urine as a method to obtain relevant information of the tumor, because of the anatomical localization of the prostate in relation to the urethra. Salido-Guadarrama et $\mathrm{al}^{60}$ obtained an AUC of 0.738 for an miR-100/200b signature in urine pellet comparing 73 patients with high-risk $\mathrm{PCa}$ and 70 patients with BPH. In our experience, a significant upregulation of miR-21, miR-141, and miR-375 was found in urinary pellets of PCa patients compared with healthy subjects, while miR214 was found significantly downregulated. ${ }^{61}$

\section{CTCs}

Tumor cells can acquire the ability to pass vessel walls and enter into the bloodstream. These cells, called CTCs, are found in circulation, which may facilitate the establishment of metastatic focus. Detection of CTCs in blood is technically quite challenging and requires highly sensitive techniques due to their relative scarcity, $\sim 1$ CTC to $10^{6}-10^{9}$ normal blood cells. ${ }^{62}$ High proportions are commonly found in advanced metastatic stages, but they are very rare in early stages. Apart from their scarcity, CTCs present a heterogeneous phenotype, as a result of intratumor heterogeneity and differences between primary tumor and metastases. ${ }^{63} \mathrm{CTCs}$ ' heterogeneity has been linked with therapy resistance and may play a role in characterizing genetic changes during cancer progression, aiding in therapeutic decisions. ${ }^{64}$

Currently, only the CellSearch ${ }^{\circledR}$ platform has obtained the approval of the FDA for monitoring of metastatic breast, colon, and $\mathrm{PCa} .{ }^{65}$ CellSearch allows the sensitive detection of CTCs by a direct method, based on immunomagnetic capture followed by a combination of positive and negative immunological selections.

Data are emerging regarding the usefulness of CTCs in $\mathrm{PCa}$, and the number of publications has increased progressively in recent years. However, there are few studies assessing CTCs in patients with localized PCa. In a review published by Maas et al, ${ }^{66}$ no sufficient evidence was found to support the prognostic value of CTC in localized PCa. The clinical utility of CTCs has been evaluated primarily for their application in patients with metastatic PCa. In advanced stages, androgen deprivation therapy (ADT) is the main strategy to slow PCa progression. Despite good initial response rates (80\%-90\%), nearly all men develop resistance to ADT. New therapies have been developed, but there is a lack of predictive biomarkers for the management of CRPC patients. In this context, the prognostic value of CTC for overall survival and disease response has been extensively evaluated, including Phase III clinical trials SWOG S0421 and COU-AA-301. Baseline CTCs' positivity ( $\geq 5$ CTCs/7.5 $\mathrm{mL}$ ) has been related with worse overall survival. ${ }^{67}$ In addition, CTCs have been proposed as a surrogate biomarker for assessment of responses to therapy in CRPC patients. ${ }^{68} \mathrm{~A}$ recent meta-analysis based on a total of 10 studies (including 1206 patients) supports these findings. ${ }^{69}$

\section{Androgen receptor}

The AR signaling axis plays a key role in both androgendependent PCa and CRPC. ${ }^{70}$ Despite initial response rates of $80 \%-90 \%$, nearly all tumors eventually regrow after a certain period developing CRPC. Some miRNAs (miR-21, miR-31, miR-34, and miR-124) were reported to target $A R$. Moreover, $A R$ can also regulate the expression of several miRNAs (miR-21, miR-27a, miR-34, miR-125b, miR-221, and let-7), and therefore affect several pathways involved in CRPC development. ${ }^{71}$ For this reason, they have been proposed as potential diagnostic markers and therapeutic targets. Furthermore, according to the researchers of the Cancer Genome Atlas Research Network, the tumors with SPOP or FOXA1 mutations have the highest levels of AR activity of all genotypically identified PCa subgroups. ${ }^{11}$

AR transcription factor is the main driver of CRPC development and of acquired resistance to drugs given for the treatment of CRPC, such as abiraterone and enzalutamide, ${ }^{72,73}$ while a minority of patients have CRPC that is non-AR driven. Molecular mechanisms behind AR reactivation in CRPC include AR gene amplification and overexpression, AR mutations, and expression of constitutively active AR variants (AR-Vs). ${ }^{74}$

AR-Vs have been frequently detected in CRPC. They are truncated $A R$ proteins lacking the AR ligand-binding domain (AR-LBD). ${ }^{75}$ Because AR-Vs contain the AR DNA-binding domain and the AR transcriptional activation domain, they can regulate transcription. Besides, AR-Vs are not regulated 
by hormonal therapies currently used in the clinic because of the lack of AR-LBD. Multiple AR-Vs derived from AR gene rearrangements and/or alternative splicing have been characterized. AR-V7 has been widely studied due to its frequency of detection in CRPC, ${ }^{76}$ as well as its potential clinical utility as a marker for treatment selection in metastatic CRPC patients. ${ }^{77}$ Patients with AR-V7 are associated with resistance to abiraterone and enzalutamide but taxane chemotherapies appear to be more efficacious in these patients. AR-V7 can be measured in both tissue and CTCs derived from CRPC patients. ${ }^{75,77}$ The test has been commercialized by Epic Sciences with the name OncotypeDX AR-V7 Nucleus Detect.

\section{PTEN}

PTEN gene is frequently mutated or deleted in several human tumors, including PCa. Data presented by the researchers of the Cancer Genome Atlas Research Network show that this gene was deleted or mutated in $17 \%$ of the PCa samples, particularly in ERG fusion-positive cases. ${ }^{11}$ Additionally, PTEN may be also inactivated by epigenetic mechanisms, such as promoter methylation ${ }^{78}$ or aberrant expression of miR-21. ${ }^{49}$ PTEN is a tumor suppressor gene located on chromosome band $10 \mathrm{q} 23$, which regulates the activity of the phosphatidylinositol 3-kinase pathway, a family of enzymes involved in cellular functions such as cell growth, differentiation, proliferation, motility, and survival.

Immunohistochemistry and fluorescence in situ hybridization (FISH) have been used to evaluate PTEN loss in formalin-fixed paraffin-embedded tissues, although FISH must be used as confirmatory assay in cases with ambiguous PTEN immunohistochemistry results or heterogeneous PTEN protein loss. ${ }^{79,80}$ PTEN loss in biopsy Gleason score 6 tumors was identified in patients with a high risk of upgrading at radical prostatectomy. ${ }^{81}$ Furthermore, a recent metaanalysis containing 10 independent cohort studies, which includes 2154 cases with positive expression of PTEN and 1006 PTEN deletion cases, showed that PTEN deletion is related to biochemical recurrence. ${ }^{82}$ Finally, PTEN loss has been related to a shorter survival in advanced $\mathrm{PCa}$ patients treated with abiraterone acetate. ${ }^{83}$

\section{Prognostic tissue biomarkers}

Historically, the Gleason score has been the most valuable predictor of PCa prognosis. Patients with a biopsy Gleason score $\geq 7$ require a radical treatment (prostatectomy or radiotherapy) because of the high risk of disease progression. In contrast, patients with a biopsy Gleason score 6 could probably have a PCa with low risk of progression, and active surveillance is the best option for them. Gleason score combined with other factors such as clinical stage, PSA serum levels, PSA density, number of positive cores, and the percentage of each core involved with cancer has been incorporated into models to select patients for active surveillance. ${ }^{84}$ However, risk of misclassification using these models is not negligible.

Novel tissue-based genomic biomarkers have been used to help in post-biopsy decision, offering additional information in risk stratification, aiding to personalize therapies. Two of these tests have been validated on men with low-risk $\mathrm{PCa}$, the Prolaris test and the Oncotype DX test, developed by Myriad Genetics (Wakara Way, Salt Lake City, UT, USA) and Genomic Health (Redwood City, CA, USA), respectively. The NCCN recommends these tests for very-low and lowrisk $\mathrm{PCa}$ in patients with at least 10 years of life expectancy to choose between active surveillance and definitive therapy.

The Prolaris test measures the aggressiveness of $\mathrm{PCa}$ evaluating a panel of 31 cell cycle progression genes related to cancer proliferation (FOXM1, CDC20, CDKN3, CDC2, KIF11, KIAA0101, NUSAP1, CENPF, ASPM, BUB1B, RRM2, DLGAP5, BIRC5, KIF20A, PLK1, TOP2A, TK1, PBK, ASF1B, C18orf24, RAD54L, PTTG1, CDCA3, MCM10, PRC1, DTL, CEP55, RAD51, CENPM, CDCA8, ORC6L) and 15 housekeeping genes (RPL38, UBA52, PSMC1, RPL4, RPL37, RPS29, SLC25A3, CLTC, TXNL1, PSMA1, RPL8, $M M A D H C, R P L 13 A$;LOC728658, PPP2CA, MRFAP1). The test is based on the results obtained by Cuzick et al ${ }^{85}$ evaluating 585 patients with clinically localized PCa diagnosed by needle biopsy between 1990 and 2003 and managed conservatively. The authors showed that the accuracy of predicting 10-year PCa-specific mortality increases adding this test to the available clinicopathological variables. Besides, the Oncotype DX test is a quantitative RT-PCR assay evaluating five housekeeping controls ( $A C T B, G A P D H, R P L P O$, $G U S B, T F R C$ ) and 12 PCa-related genes representing four biological pathways with a role in prostate tumorigenesis: androgen signaling (AZGP1, FAM13C1, KLK2, SRD5A2), cell proliferation (TPX2), cellular organization (FLNC, GSN, GSTM2, TPM2), and stromal response (BGN, COL1A1, $S F R P 4)$. The test offers a genomic prostate score related with the probability of recurrence after radical prostatectomy and adverse pathology (primary Gleason pattern 4 or any pattern 5 and/or pT3 disease) at surgery. ${ }^{86}$

On the other hand, the ProMark ${ }^{\circledR}$ test is a multiplex immunofluorescent signature developed by Metamark (Cambridge, MA, USA), which involves eight proteins that provide information about the aggressiveness of tumor. ${ }^{87}$ 
This test is also recommended by NCCN for very-low and low-risk PCa in patients with at least 10 years' life expectancy to choose the more appropriate treatment. The biomarkers included in this test were selected evaluating their ability to equally predict aggressive prostate pathology and lethal outcome regardless of whether they were measured in low- or high-grade cancer regions from each patient. This procedure ensures the robustness of the test to errors related to biopsy sampling variation that occurs because of PCa heterogeneity. ${ }^{88}$ This approach suggests that ProMark test could be used to distinguish low-risk from high-risk PCa in biopsy samples with high accuracy. However, at the moment, the test has not been validated for this use.

\section{Conclusion}

The available genomic and proteomic assays previously described could improve the predictive value over other PCa risk classification systems based on clinicopathologic variables. Nevertheless, further studies are necessary to validate these tests in order to aid in personalized treatment decisions. In contrast, the novel serum and urine biomarkers are used to select patients for biopsy. The published results showed that PCA3 score, PHI, and 4Kscore can reduce the number of unnecessary biopsies, outperforming total PSA and \%fPSA. Besides, several reports documented their role to repeat biopsy in patients with a previous negative result. Actually, persistent increases in PSA serum levels in patients with previous negative biopsy remain a clinical dilemma. Approximately $75 \%$ of patients with PSA between 2 and $10 \mu \mathrm{g} / \mathrm{L}$ have a negative result in the biopsy. Nevertheless, a significant false-negative detection rate must be considered because sampling errors are frequent, involving 10\%-20\% of clinically significant $\mathrm{PCa} .{ }^{89}$ Strategies to decide when and in which cases repetition of biopsy is necessary are required. Published data suggest that new emerging biomarkers could also improve the accuracy of total PSA and \%fPSA in predicting the presence of $\mathrm{PCa}$ at repeated biopsy. Besides, PHI and 4 Kscore provide with high accuracy an individual risk of high-grade PCa. In contrast, the relationship of PCA3 score with tumor aggressiveness remains unclear, with studies showing opposite results.

On the other hand, although screening studies to select miRNA or gene expression profiles are laborious and expensive, the technological innovations and bioinformatics approaches make these studies more viable. Bioinformatics tools and the development of next-generation sequencing technologies have enabled the identification of novel genomic profiles and their clinical application for PCa management. Furthermore, improvement in methodology is required to better evaluate miRNAs and circulating tumor DNA in PCa patients, despite the fact that preliminary results are promising.

To conclude, emerging biomarkers could change the management of early $\mathrm{PCa}$, offering more accurate results than current biomarkers. Nonetheless, large prospective studies comparing these new emerging biomarkers among them are required to know their real value in $\mathrm{PCa}$ detection and prognosis.

\section{Disclosure}

The authors report no conflicts of interest in this work.

\section{References}

1. Ferlay J, Soerjomataram I, Dikshit R, et al. Cancer incidence and mortality worldwide: sources, methods and major patterns in GLOBOCAN 2012. Int J Cancer. 2015;136(5):E359-E386.

2. Siegel RL, Miller KD, Jemal A. Cancer statistics, 2018. CA Cancer J Clin. 2018;68(1):7-30.

3. Klotz L. Low-risk prostate cancer can and should often be managed with active surveillance and selective delayed intervention. Nat Clin Pract Urol. 2008;5(1):2-3.

4. Tosoian JJ, Carter HB, Lepor A, Loeb S. Active surveillance for prostate cancer: current evidence and contemporary state of practice. Nat Rev Urol. 2016;13(4):205-215.

5. D'Amico AV, Whittington R, Malkowicz SB, et al. Clinical utility of the percentage of positive prostate biopsies in defining biochemical outcome after radical prostatectomy for patients with clinically localized prostate cancer. J Clin Oncol. 2000;18(6):1164-1172.

6. Brajtbord JS, Leapman MS, Cooperberg MR. The CAPRA score at 10 years: contemporary perspectives and analysis of supporting studies. Eur Urol. 2017;71(5):705-709.

7. NCCN Prostate Cancer Guideliness [webpage on the Internet]. Version 2. 2017. Available from: https://www.nccn.org/professionals/physician_gls/pdf/prostate_core.pdf. Accessed March 15, 2018.

8. Lepor H, Donin NM. Gleason 6 prostate cancer: serious malignancy or toothless lion? Oncology (Williston Park). 2014;28(1):16-22.

9. Rubin MA, Girelli G, Demichelis F. Genomic correlates to the newly proposed grading prognostic groups for prostate cancer. Eur Urol. 2016;69(4):557-560.

10. Lapointe J, Li C, Higgins JP, et al. Gene expression profiling identifies clinically relevant subtypes of prostate cancer. Proc Natl Acad Sci USA. 2004;101(3):811-816.

11. Cancer Genome Atlas Research Network. The molecular taxonomy of primary prostate cancer. Cell. 2015;163(4):1011-1025.

12. Lee R, Localio AR, Armstrong K, Malkowicz SB, Schwartz JS. A metaanalysis of the performance characteristics of the free prostate-specific antigen test. Urology. 2006;67(4):762-768.

13. Filella X, Giménez N. Evaluation of [-2] proPSA and Prostate Health Index (PHI) for the detection of prostate cancer: a systematic review and meta-analysis. Clin Chem Lab Med. 2013;51(4):729-739.

14. Bruzzese D, Mazzarella C, Ferro M, et al. Prostate health index vs. percent free prostate-specific antigen for prostate cancer detection in men with "gray" prostate-specific antigen levels at first biopsy: systematic review and meta-analysis. Transl Res. 2014;164(6):444-451.

15. Filella X, Foj L, Alcover J, Augé JM, Molina R, Jiménez W. The influence of prostate volume in prostate health index performance in patients with total PSA lower than $10 \mu \mathrm{g} / \mathrm{L}$. Clin Chim Acta. 2014;436:303-307. 
16. Tosoian JJ, Druskin SC, Andreas D, et al. Prostate Health Index density improves detection of clinically significant prostate cancer. BJU Int. 2017;120(6):793-798.

17. Filella X, Foj L, Augé JM, Molina R, Alcover J. Clinical utility of $\%$ p2PSA and prostate health index in the detection of prostate cancer. Clin Chem Lab Med. 2014;52(9):1347-1355.

18. De la Calle C, Patil D, Wei JT, et al. Multicenter evaluation of the Prostate Health Index to detect aggressive prostate cancer in biopsy naïve men. J Urol. 2015;194(1):65-72.

19. Filella X, Foj L. Emerging biomarkers in the detection and prognosis of prostate cancer. Clin Chem Lab Med. 2015;53(7):963-973.

20. Parekh DJ, Punnen S, Sjoberg DD, et al. A multi-institutional prospective trial in the USA confirms that the 4 Kscore accurately identifies men with high-grade prostate cancer. Eur Urol. 2015(3);68:464-470.

21. Braun K, Sjoberg DD, Vickers AJ, Lilja H, Bjartell AS. A four-kallikrein panel predicts high-grade cancer on biopsy: independent validation in a community cohort. Eur Urol. 2016;69(3):505-511.

22. Stattin P, Vickers AJ, Sjoberg DD, et al. Improving the specificity of screening for lethal prostate cancer using prostate-specific antigen and a panel of kallikrein markers: a nested case-control study. Eur Urol. 2015;68(2):207-213.

23. Lin DW, Newcomb LF, Brown MD, et al; Canary Prostate Active Surveillance Study Investigators. Evaluating the Four Kallikrein Panel of the $4 \mathrm{Kscore}$ for prediction of high-grade prostate cancer in men in the Canary Prostate Active Surveillance Study. Eur Urol. 2017;72(3):448-454.

24. Peracaula R. Altered glycosylation pattern allows the distinction between prostate-specific antigen (PSA) from normal and tumor origins. Glycobiology. 2003;13(6):457-470.

25. Meany DL, Zhang Z, Sokoll LJ, Zhang H, Chan DW. Glycoproteomics for prostate cancer detection: changes in serum PSA glycosylation patterns. J Proteome Res. 2009;8(2):613-619.

26. Ohyama C, Hosono M, Nitta K, et al. Carbohydrate structure and differential binding of prostate specific antigen to Maackia amurensis lectin between prostate cancer and benign prostate hypertrophy. Glycobiology. 2004;14(8):671-679.

27. Llop E, Ferrer-Batallé M, Barrabés S, et al. Improvement of prostate cancer diagnosis by detecting PSA glycosylation-specific changes. Theranostics. 2016;6(8):1190-1204.

28. Kyselova Z, Mechref Y, Al Bataineh MM, et al. Alterations in the serum glycome due to metastatic prostate cancer. J Proteome Res. 2007;6(5):1822-1832.

29. Li QK, Chen L, Ao M-H, et al. Serum fucosylated prostate-specific antigen (PSA) improves the differentiation of aggressive from nonaggressive prostate cancers. Theranostics. 2015;5(3):267-276.

30. Bussemakers MJ, van Bokhoven A, Verhaegh GW, et al. DD3: a new prostate-specific gene, highly overexpressed in prostate cancer. Cancer Res. 1999;59(23):5975-5979.

31. Clarke RA, Zhao Z, Guo AY, et al. New genomic structure for prostate cancer specific gene PCA3 within BMCC1: implications for prostate cancer detection and progression. PLoS One. 2009;4(3):e4995.

32. Filella X, Foj L, Milà M, Augé JM, Molina R, Jiménez W. PCA3 in the detection and management of early prostate cancer. Tumour Biol. 2013;34(3):1337-1347.

33. Cui Y, Cao W, Li Q, et al. Evaluation of prostate cancer antigen 3 for detecting prostate cancer: a systematic review and meta-analysis. Sci Rep. 2016;6:25776.

34. Roobol MJ, Schröder FH, van Leeuwen P, et al. Performance of the prostate cancer antigen 3 (PCA3) gene and prostate-specific antigen in prescreened men: exploring the value of PCA3 for a first-line diagnostic test. Eur Urol. 2010;58(4):475-481.

35. Schröder FH, Venderbos LD, van den Bergh RC, et al. Prostate cancer antigen 3: diagnostic outcomes in men presenting with urinary prostate cancer antigen 3 scores $\geq 100$. Urology. 2014;83(3):613-616.

36. Merola R, Tomao L, Antenucci A, et al. PCA3 in prostate cancer and tumor aggressiveness detection on 407 high-risk patients: a National Cancer Institute experience. J Exp Clin Cancer Res. 2015;34:15.
37. Chevli KK, Duff M, Walter P, et al. Urinary PCA3 as a predictor of prostate cancer in a cohort of 3,073 men undergoing initial prostate biopsy. J Urol. 2014;191(6):1743-1748.

38. Haese, A, de la Taille A, van Poppel H, et al. Clinical utility of the PCA3 urine assay in European men scheduled for repeat biopsy. Eur Urol. 2008;54(5):1081-1088.

39. Hessels D, Van Gils MP, Van Hooij O, et al. Predictive value of PCA3 in urinary sediments in determining clinico-pathological characteristics of prostate cancer. Prostate. 2010;70(1):10-16.

40. Foj L, Milà M, Mengual L, et al. Real-time PCR PCA3 assay is a useful test measured in urine to improve prostate cancer detection. Clin Chim Acta. 2014;435:53-58

41. Tomlins SA, Rhodes DR, Perner S, et al. Recurrent fusion of TMPRSS2 and ETS transcription factor genes in prostate cancer. Science. 2005;310(5748):644-648

42. Leyten GH, Hessels D, Jannink SA, et al. Prospective multicenter evaluation of PCA3 and TMPRSS2-ERG gene fusions as diagnostic and prognostic urinary biomarkers for prostate cancer. Eur Urol. 2014;65(3):534-542.

43. Tomlins SA, Day JR, Lonigro RJ, et al. Urine TMPRSS2:ERG Plus PCA3 for individualized prostate cancer risk assessment. Eur Urol. 2016;70(1):45-53

44. McKiernan J, Donovan MJ, O’Neill V, et al. A novel urine exosome gene expression assay to predict high-grade prostate cancer at initial biopsy. JAMA Oncol. 2016;2(7):882-889.

45. Esteller M. Non-coding RNAs in human disease. Nat Rev Genet 2011;12(12):861-874.

46. Lee RC, Feinbaum RL, Ambros V. The C. elegans heterochronic gene lin-4 encodes small RNAs with antisense complementarity to lin-14. Cell. 1993;75(5):843-854.

47. Liu C, Kelnar K, Liu B, et al. The microRNA miR-34a inhibits prostate cancer stem cells and metastasis by directly repressing CD44. Nat Med. 2011;17(2):211-215.

48. Kong D, Heath E, Chen W, et al. Loss of let-7 up-regulates EZH2 in prostate cancer consistent with the acquisition of cancer stem cell signatures that are attenuated by BR-DIM. PLoS One. 2012;7(3):e33729.

49. M. Folini, P. Gandellini, N. Longoni, et al. miR-21: an oncomir on strike in prostate cancer. Mol Cancer. 2010, 9:12.

50. Cimmino A, Calin GA, Fabbri M, et al. miR-15 and miR-16 induce apoptosis by targeting BCL2. Proc Natl Acad Sci USA. 2005;102(39): 13944-13949.

51. Verdoodt B, Neid M, Vogt M, et al. MicroRNA-205, a novel regulator of the anti-apoptotic protein $\mathrm{Bcl} 2$, is downregulated in prostate cancer. Int J Oncol. 2013;43(1):307-314.

52. Hagman Z, Larne O, Edsjö A, et al. miR-34c is down regulated in prostate cancer and exerts tumor suppressive functions. Int J Cancer. 2010;127(12):2768-2776.

53. Kalluri K, Weinberg RA. The basics of epithelial-mesenchymal transition. J Clin Invest. 2009;119(6):1420-1428.

54. Gregory PA, Bert AG, Paterson EL, et al. The miR-200 family and miR-205 regulate epithelial to mesenchymal transition by targeting ZEB1 and SIP1. Nat Cell Biol. 2008;10(5):593-601.

55. Vandewalle C, Comijn J, De Craene B, et al. SIP1/ZEB2 induces EMT by repressing genes of different epithelial cell-cell junctions. Nucleic Acids Res. 2005;33(20):6566-6578.

56. Coppola V, Musumeci M, Patrizii M, et al. BTG2 loss and miR-21 upregulation contribute to prostate cell transformation by inducing luminal markers expression and epithelial-mesenchymal transition. Oncogene. 2013;32(14):1843-1853.

57. Filella X, Foj L. miRNAs as novel biomarkers in the management of prostate cancer. Clin Chem Lab Med. 2017;55(5):715-736.

58. Mihelich BL, Maranville JC, Nolley R, Peehl DM, Nonn L. Elevated serum microRNA levels associate with absence of high-grade prostate cancer in a retrospective cohort. PLoS One. 2015;10(4):e0124245.

59. Al-Qatati A, Akrong C, Stevic I, et al. Plasma microRNA signature is associated with risk stratification in prostate cancer patients. Int $J$ Cancer. 2017;141(6):1231-1239. 
60. Salido-Guadarrama AI, Morales-Montor JG, Rangel-Escareño C, et al. Urinary microRNA-based signature improves accuracy of detection of clinically relevant prostate cancer within the prostate-specific antigen grey zone. Mol Med Rep. 2016;13(6):4549-4560.

61. Foj L, Ferrer F, Serra M, et al. Exosomal and non-exosomal urinary miRNAs in prostate cancer detection and Prognosis. Prostate. 2017;77(6):573-583.

62. Zieglschmid V, Hollmann C, Böcher O. Detection of disseminated tumor cells in peripheral blood. Crit Rev Clin Lab Sci. 2005;42(2):155-196.

63. Turajlic S, Swanton C. Metastasis as an evolutionary process. Science. 2016;352(6282):169-175.

64. Scher HI, Graf RP, Schreiber NA, et al. Phenotypic heterogeneity of circulating tumor cells informs clinical decisions between AR signaling inhibitors and taxanes in metastatic prostate cancer. Cancer Res. 2017;77(20):5687-5698.

65. Liberko M, Kolostova K, Bobek V. Essentials of circulating tumor cells for clinical research and practice. Crit Rev Oncol Hematol. 2013;88(2):338-356.

66. Maas M, Hegemann M, Rausch S, Bedke J, Stenzl A, Todenhöfer T. Circulating tumor cells and their role in prostate cancer. Asian JAndrol. Epub 2017 Aug 22.

67. Goldkorn A, Ely B, Quinn DI, et al. Circulating tumor cell counts are prognostic of overall survival in SWOG S0421: a Phase III trial of docetaxel with or without atrasentan for metastatic castration-resistant prostate cancer. J Clin Oncol. 2014;32(11):1136-1142.

68. Scher HI, Heller G, Molina A, et al. Circulating tumor cell biomarker panel as an individual-level surrogate for survival in metastatic castration-resistant prostate cancer. J Clin Oncol. 2015;33(12):1348-1355.

69. Zheng Y, Zhang C, Wu J, et al. Prognostic value of circulating tumor cells in castration resistant prostate cancer: a meta-analysis. Urol J. 2016;13(6):2881-2888.

70. Heinlein CA, Chang C. Androgen receptor in prostate cancer. Endocr Rev. 2004;25(2):276-308.

71. Song C, Huan C, Xu C, Ru G. Uncovering the roles of miRNAs and their relationship with androgen receptor in prostate cancer. IUBMB Life. 2014;66(6):379-386.

72. Schrader AJ, Boegemann M, Ohlmann CH, et al. Enzalutamide in castration-resistant prostate cancer patients progressing after docetaxel and abiraterone. Eur Urol. 2014;65(1):30-36.

73. Noonan KL, North S, Bitting RL, Armstrong AJ, Ellard SL, Chi KN. Clinical activity of abiraterone acetate in patients with metastatic castration-resistant prostate cancer progressing after enzalutamide. Ann Oncol. 2013;24(7):1802-1807.

74. Karantanos T, Evans CP, Tombal B, Thompson TC, Montironi R, Isaacs WB. Understanding the mechanisms of androgen deprivation resistance in prostate cancer at the molecular level. Eur Urol. 2015;67(3):470-479.
75. Antonarakis ES, Armstrong AJ, Dehm SM, Luo J. Androgen receptor variant-driven prostate cancer: clinical implications and therapeutic targeting. Prostate Cancer Prostatic Dis. 2016;19(3):231-241.

76. Robinson D, Van Allen EM, Wu YM, et al. Integrative clinical genomics of advanced prostate cancer. Cell. 2015;161(5):1215-1228.

77. Luo J. Development of AR-V7 as a putative treatment selection marker for metastatic castration-resistant prostate cancer. Asian J Androl. 2016;18(4):580-585.

78. Whang YE, Wu X, Suzuki H, et al. Inactivation of the tumor suppressor PTEN/MMAC1 in advanced human prostate cancer through loss of expression. Proc Natl Acad Sci USA. 1998;95(9):5246-5250.

79. Lotan TL, Wei W, Ludkovski O, et al. Analytic validation of a clinicalgrade PTEN immunohistochemistry assay in prostate cancer by comparison with PTEN FISH. Mod Pathol. 2016;29(8):904-914.

80. Cuzick J, Yang ZH, Fisher G, et al; Transatlantic Prostate Group. Prognostic value of PTEN loss in men with conservatively managed localised prostate cancer. Br J Cancer. 2013;108(12):2582-2589.

81. Lotan TL, Carvalho FLF, Peskoe SB, et al. PTEN loss is associated with upgrading of prostate cancer from biopsy to radical prostatectomy. Mod Pathol. 2015;28(1):128-137.

82. Xie H, Xie B, Liu C, Wang J, Xu Y. Association of PTEN expression with biochemical recurrence in prostate cancer: results based on previous reports. Onco Targets Ther. 2017;10:5089-5097.

83. Ferraldeschi R, Nava Rodrigues D, Riisnaes R, et al. PTEN protein loss and clinical outcome from castration-resistant prostate cancer treated with abiraterone acetate. Eur Urol. 2015;67(4):795-802.

84. Syed JS, Javier-Desloges J, Tatzel S, et al. Current management strategy for active surveillance in prostate cancer. Curr Oncol Rep. 2017;19(2):11.

85. Cuzick J, Stone S, Fisher G, et al. Validation of an RNA cell cycle progression score for predicting death from prostate cancer in a conservatively managed needle biopsy cohort. Br J Cancer. 2015;113(3): 382-389.

86. Cullen J, Rosner IL, Brand TC, et al. A biopsy-based 17-gene genomic prostate score predicts recurrence after radical prostatectomy and adverse surgical pathology in a racially diverse population of men with clinically low- and intermediate-risk prostate cancer. Eur Urol. 2015;68(1):123-131.

87. Blume-Jensen P, Berman DM, Rimm DL, et al. Development and clinical validation of an in situ biopsy-based multimarker assay for risk stratification in prostate cancer. Clin Cancer Res. 2015;21(11):2591-2600.

88. Shipitsin M, Small C, Choudhury S, et al. Identification of proteomic biomarkers predicting prostate cancer aggressiveness and lethality despite biopsy-sampling error. Br J Cancer. 2014;111(6):1201-1212.

89. Capitanio U, Pfister D, Emberton M. Repeat prostate biopsy: rationale, indications, and strategies. Eur Urol Focus. 2015;1(2):127-136.
Pharmacogenomics and Personalized Medicine

\section{Publish your work in this journal}

Pharmacogenomics and Personalized Medicine is an international, peerreviewed, open access journal characterizing the influence of genotype on pharmacology leading to the development of personalized treatment programs and individualized drug selection for improved safety, efficacy and sustainability. This journal is indexed on the American Chemical

\section{Dovepress}

Society's Chemical Abstracts Service (CAS). The manuscript management system is completely online and includes a very quick and fair peer-review system, which is all easy to use. Visit http://www.dovepress. $\mathrm{com} /$ testimonials.php to read real quotes from published authors. 